\title{
Experimental validation of a 2D-slit homogenizer for space based imaging spectrometers
}

\section{Timon Hummel, Claude Coatantiec, Xavier Gnata, Tobias Lamour, Rémi Rivière, et al.}

Timon Hummel, Claude Coatantiec, Xavier Gnata, Tobias Lamour, Rémi Rivière, Christian Meister, Jasper Krauser, Dennis Weise, Mark Wenig, "Experimental validation of a 2D-slit homogenizer for space based imaging spectrometers," Proc. SPIE 11852, International Conference on Space Optics - ICSO 2020, 118520L (11 June 2021); doi: 10.1117/12.2599167 SPIE. Event: International Conference on Space Optics - ICSO 2021, 2021, Online 


\section{International Conference on Space Optics-ICSO 2020}

Virtual Conference

30 March-2 April 2021

Edited by Bruno Cugny, Zoran Sodnik, and Nikos Karafolas
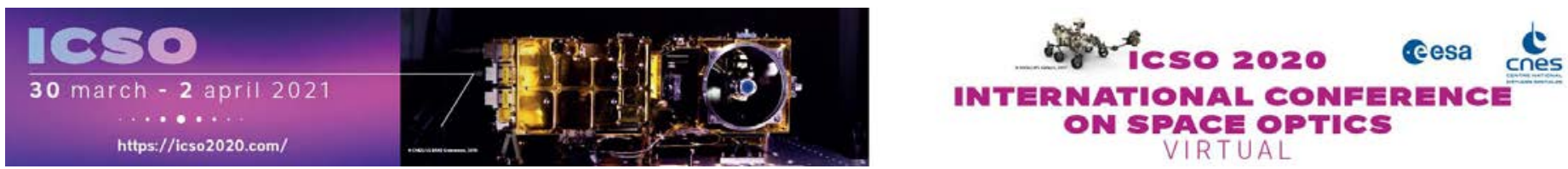

\section{Experimental validation of a 2D-Slit homogenizer for space based imaging spectrometers}

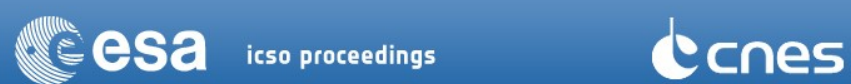




\title{
Experimental validation of a 2D-Slit homogenizer for space based imaging spectrometers
}

\author{
Timon Hummel ${ }^{\mathrm{a}, \mathrm{d}}$, Claude Coatantiec ${ }^{\mathrm{b}}$, Xavier Gnata ${ }^{\mathrm{b}}$, Tobias Lamour ${ }^{\mathrm{c}}$, Remi Riviere , $^{\mathrm{a}}$ \\ Christian Meister ${ }^{\mathrm{a}}$, Jasper Krauser ${ }^{\mathrm{a}}$, Dennis Weise $^{\mathrm{c}}$, and Mark Wenig ${ }^{\mathrm{d}}$ \\ ${ }^{a}$ Airbus Defence and Space, Robert-Koch-Str.1, 82024 Taufkirchen, Germany \\ ${ }^{\mathrm{b}}$ Airbus Defence and Space, 31 rue des Cosmonautes, 31402 Toulouse, France \\ ${ }^{\mathrm{c}}$ Airbus Defence and Space, Claude-Dornier-Str., 88090 Immenstaad, Germany \\ dMeterological Institute Munich - Ludwig-Maximilians-Universität, Theresienstraße 37, 80333 \\ Munich, Germany
}

\begin{abstract}
The analysis of recent Earth observation spectrometer missions revealed the impact of spatially heterogeneous Earth radiance scenes on the spectral accuracy of the instruments. One of the most critical observations is the distortion of the instrument spectral response function (ISRF) induced by radiometric contrast in the Earth radiance scene. In order to meet the high precision and accuracy of quantifying the spatial distribution of the atmospheric composition, stringent requirements on the ISRF knowledge are defined such as shape stability, centroid position of the spectral channel centre and the Full Width at Half Maximum (FWHM).

In the framework of the CO2M A/B1 study, Airbus investigated a new slit concept called 2D-slit homogenizer (2DSH) for the mitigation of spatially non-uniform scenes. This is done by replacing the classical spectrometer slit with non-circular core multimode fibres, which scramble the light in along-track (ALT) and across-track (ACT) direction and hence average the contrast of the Earth scene in both dimensions. The final 2DSH will be made of several adjoined fibres, assembled in a bundle. A single fibre core dimension defines the spectral extent of the slit (ALT) and the minimum achievable spatial sample (ACT). Consequently, the full swath width covered by the instrument is given by the total size of the fibre bundle in ACT.

Here, we present an experimental validation of the 2DSH in terms of scrambling efficiency and radiometry. In order to probe the fibre characteristics for non-uniform scenes, we designed and constructed a setup which allows us to track and tune multiple high contrast scene cases as a fibre input facet illumination. The scrambling efficiency performance of the fibre is assessed by measuring the near- and far-field intensity distribution of light transmitted through the fibre for different scene cases. Furthermore, we analyze the impact of focal ratio degradation in terms of radiometric losses and compare the results in the NIR and SWIR wavelength for different stress cases on the fibre.
\end{abstract}

Keywords: Earth observation, Slit homogenizer, Spectrometer, Remote Sensing

\section{INTRODUCTION}

The impact of heterogeneous scenes on the spectral accuracy of space-borne imaging spectrometer was recognized and discussed in several recent Earth observation missions, such as the Ozone Monitoring Instrument (OMI) ${ }^{1}$ Sentinel- $4,{ }^{2}$ TROPOMI ${ }^{3,4}$ and Sentinel-5/UVNS. ${ }^{5,6}$ In such space-borne imaging spectrometers, the Earth radiance scene gets imaged by the telescope onto the instrument entrance slit plane. In the subsequent spectrograph, the slit illumination gets spectrally resolved by a dispersive element and re-imaged on the focal plane array (FPA) by imaging optics. The slit image for a monochromatic wavelength on the detector is called the instrument spectral response function (ISRF). It is given by a convolution of the slit illumination, the optical point spread function (PSF) of the spectrometer imaging optics and the FPA pixel characteristics. As the slit illumination is directly related to the observed Earth scene, a scene heterogeneity also results in a non-uniform

Further author information: (Send correspondence to Timon Hummel)

Timon Hummel: E-mail: timon.hummel@airbus.com, Telephone: +49 8931793793 
slit illumination. Consequently, the shape of the ISRF varies with the observed scene. As the ISRF is the direct link between the radiative transfer model and the observed spectrum by the instrument, a scene dependent ISRF will have an immediate impact on the quality of the trace gas retrieval products.

The $\mathrm{CO}_{2}$ Monitoring Mission $(\mathrm{CO} 2 \mathrm{M})$ aims to quantify sources and sinks of carbon dioxide $\left(\mathrm{CO}_{2}\right)$ and methane $\left(\mathrm{CH}_{4}\right)$ by measuring their distribution in the atmosphere. In order to meet the retrieval precision and accuracy goals, several spectral requirements are formulated in the stability of the ISRF under the condition of heterogeneous scenes. For a sharp contrast scene, which corresponds to a sudden transition from bright to dark slit illumination in the middle of a spectral sample, the ISRF in-flight shape knowledge has to be better than $1.5 \%$ and the position of the spectral centre channel shall be known with accuracy better than $0.002 \mathrm{~nm}$ (NIR).

In the framework of the CO2M A/B1 study, we investigated a new slit concept called 2D-Slit Homogenizer (2DSH). This slit concept replaces the classical spectrometer slit with non-circular core multimode fibres, which scramble the light in across-track (ACT) and along-track (ALT) direction. The dimension of each rectangular fibre core represents a single spatial and spectral sample of the instrument. The along-slit dimension of the fibre defines the spectral extent of the slit and the ACT dimension represents the minimum spatial sample. The full swath width of the instrument is given by the total size of the fibres across the slit.

In this contribution, we investigate the scrambling performance of a non-circular fibre-based slit for different Earth scenes and particularly discuss the near field (NF) and far field (FF) homogeneity after the fibre. Further, we assess the radiometric losses due to focal ratio degradation (FRD).

\section{OPTICAL SETUP}

The purpose of the setup is to assess the scrambling performance of the fibre and the radiometric losses due to FRD. For the tests conducted in this paper, we used a NIR- $(780 \mathrm{~nm})$ and SWIR $(1550 \mathrm{~nm})$ laser source. Figure (2) shows the experimental setup of the measurements. The first part of the setup consists of an illumination system, which is used to simulate a homogeneous telescope pupil and a tunable Earth scene. A two-dimensionally adjustable slit at the output port of the integrating sphere defines the shape and size of the emitted beam. The slit itself is re-imaged again onto the fibre entrance plane, representing the Earth scene. An adjustable aperture in the telescope pupil plane is used to set the F-number $(F \#)$ of the beam which is fed into the fibre. A beamsplitter in the telescope pupil plane allows to track the back reflection of the fibre input, which gets imaged using an additional camera. This allows to precisely track and tune the applied fibre input illumination. After the fibre, we re-image the fibre NF output by a microscope objective with $\mathrm{x} 4$ magnification. The FF is directly measured by placing a camera at several centimetre distance after the fibre output port, where the exact camera position is controlled by a 3D-motorized translation stage. The setup is depicted in Fig. (2). In the frame of this study, we tested two different lengths of rectangular core fibres provided by CeramOptec. They share the same core dimension of $300 \times 100 \mu m \pm 2 \%$ (ACT/ALT) and a cladding of $180 \times 350 \mu m \pm 2 \%$ (ACT/ALT). The fibres are custom made by CeramOptec and have their outer coating remove on the fibre tips to minimise the blind gap. The first set of fibres has a length of $50 \mathrm{~mm} \pm 3 \mathrm{~mm}$ and the second set of $100 \mathrm{~cm} \pm 5 \mathrm{~mm}$.
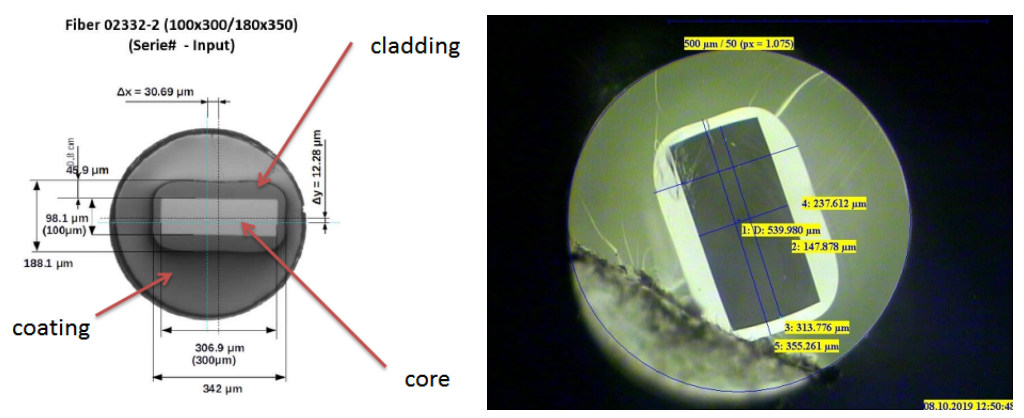

Figure 1: (Left) Commercial off-the-shelf rectangular fibre by CeramOptec. (Right) Custom fibre by CeramOptec with the outer coating removed on the fibre tips to minimise the blind gap. 


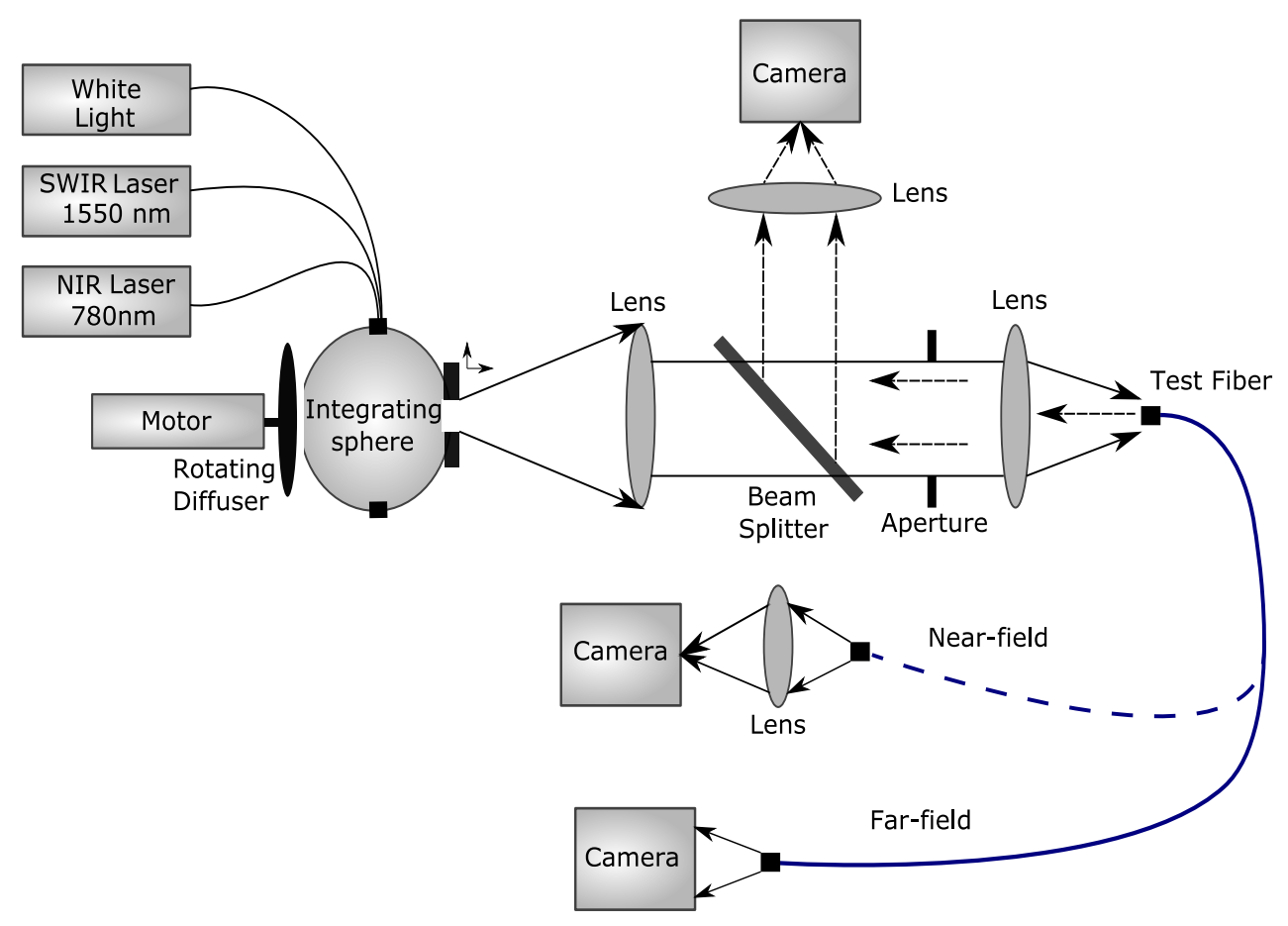

Figure 2: Schematic presentation of the fibre-test setup to measure the near and far field intensity distribution of the fibre output.

\section{NEAR-FIELD AND FAR-FIELD INTENSITY DISTRIBUTION}

The ISRF is given by the convolution of the geometrical image of the slit on the FPA, the spectrograph PSF and the detector pixel characteristics. In order to test the impact of non-uniform illumination at the entrance of the 2DSH on the ISRF stability, we assess the scrambling performance of the fibre in the NF. Moreover, the spectrograph PSF is the Fourier transform of the light distribution in the spectrograph pupil plane. Therefore, if the far field illumination at the exit of the fibre is a function of the illumination pattern at the entrance of the fibre, then the ISRF in the context of heterogeneous scenes will also be affected due to PSF variations. The strength of this effect is mainly driven by the geometrical optical aberrations present in the spectrograph.

\subsection{Near-Field}

In order to test the NF scrambling performance, we illuminated the fibre with a high-contrast scene in ALT, where $50 \%$ of the fibre entrance is illuminated. Note that these scenes are not representative of real scenarios for a push-broom spectrometer with finite integration time and finite FoV. During the integration time, the scene contrast will be smeared over the entrance slit due to the movement of the platform. However, such scenes are conveniently used in experimental measurements. We repeated the test for a fibre length of $50 \mathrm{~mm}$ and $100 \mathrm{~cm}$. Figure (3) shows the fibre input illumination and the respective fibre output in the NF for the short fibre. The cross-section of the fibre output intensity distributions keeps a prominent residual gradient depending on the symmetry of the fibre input illumination. This will directly translate into ISRF error in terms of shape and centroid. We repeated the measurement for the long fibre $(100 \mathrm{~cm})$ and the results are shown in Fig (4). The homogenization of the fibre output is significantly better compared with the short fibre. Therefore we conclude that the fibre length is a crucial parameter for the scrambling performance of a $2 \mathrm{DSH}$. 


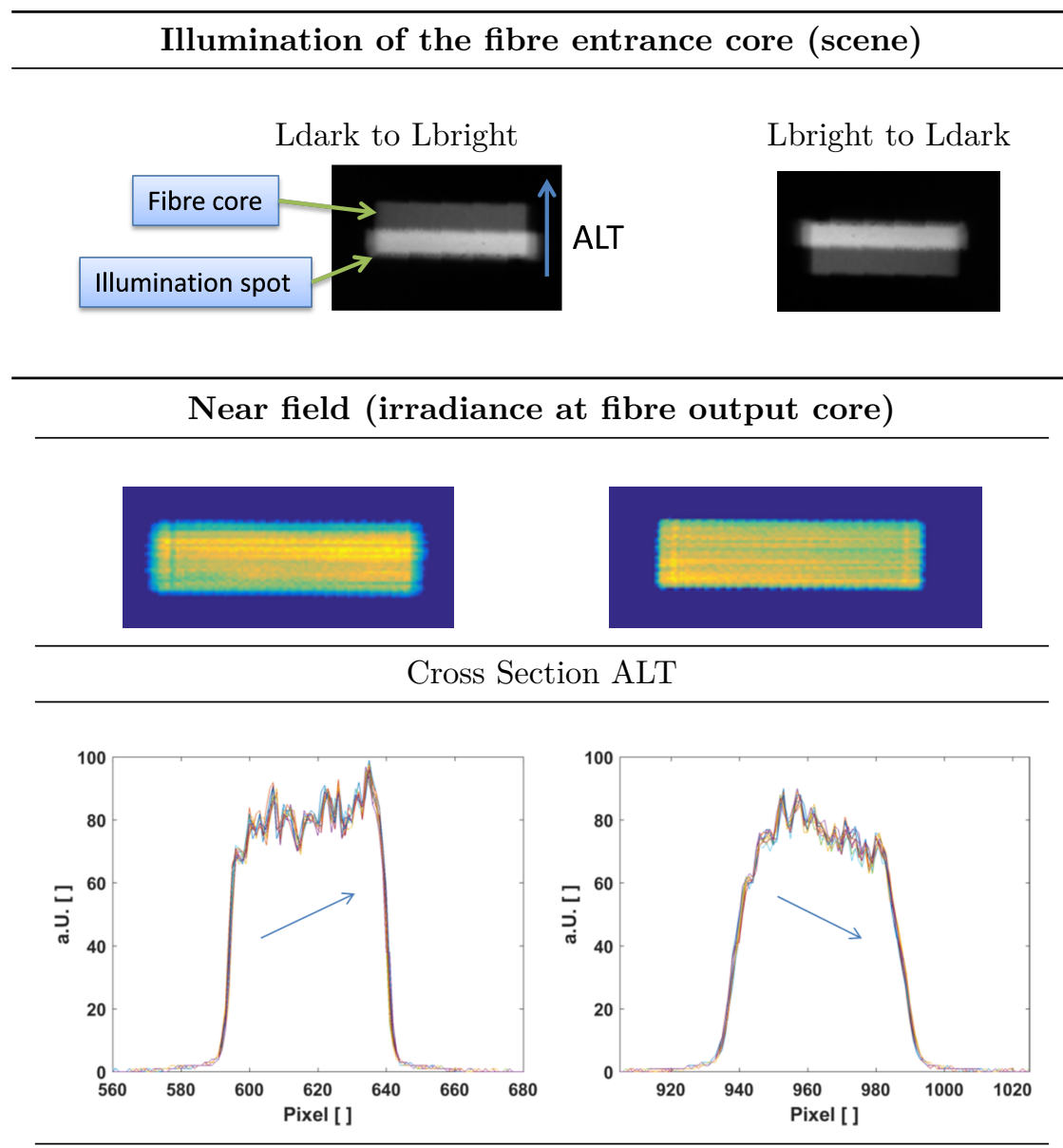

Figure 3: Short fibre NF scrambling for non-uniform scenes in ALT.
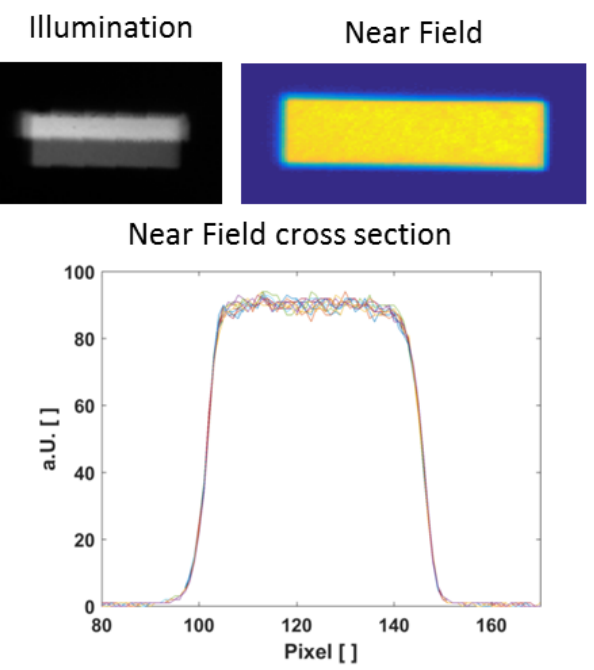

Figure 4: Long fibre NF scrambling for non-uniform scenes in ALT. 


\subsection{Far Field}

In space-based imaging spectrometers equipped with a classical slit, which is acting as a field stop, the telescope pupil intensity distribution is, besides some diffraction edge effects in the slit plane, preserved in the spectrograph pupil. However, when employing a fibre-based 2DSH, the spectrograph pupil will be altered with respect to the telescope pupil, depending on the input illumination of the fibre. Figure (5) shows the FF intensity distribution for the $100 \mathrm{~cm}$ long fibre. We applied a fibre illumination of $20 \%$ and moved the illumination spot in the ACT direction of the fibre. The resulting FF intensity profiles for the two input illuminations have symmetric shapes, which follow an inverse relationship depending on the illumination of the fibre entrance. Depending on the position of the illumination, the FF intensity pattern has either a peak or a dip in the central spot. The general behaviour of the FF was observed with monochromatic light sources (NIR and SWIR) as well as a broadband source.

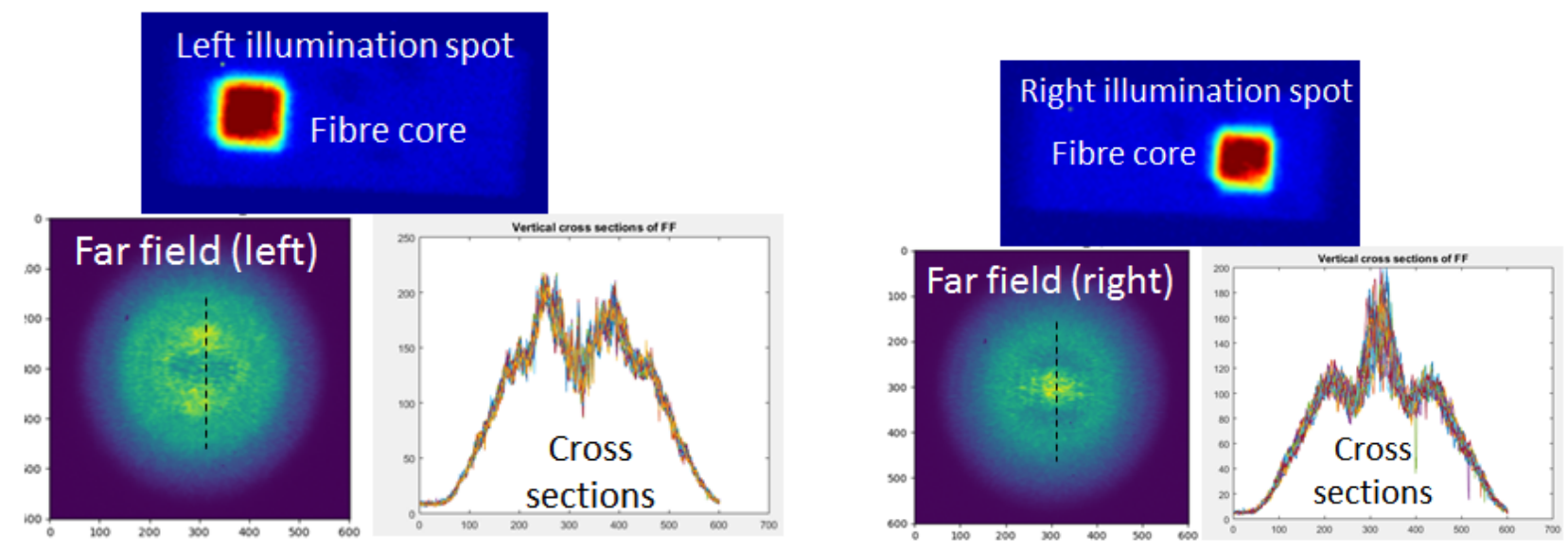

Figure 5: Fibre FF intensity distribution obtained from a $100 \mathrm{~cm}$ fibre for two different antisymmetric fibre inputs. The FF intensity pattern have symmetric shapes with either a peak or a dip in the centre, depending on the location of the fibre input. The cross-section is plotted along the ALT direction.

The scene dependent spectrograph pupil intensity distribution will alter the spectrograph PSF on the FPA and hence the ISRF shape and centroid stability. We quantify the FF impact on the ISRF by propagating the experimentally acquired FF intensity distribution from Fig.(5) through a CodeV model of the Airbus CO2M A/B1 design. The ISRF errors have been computed for three wavelengths of the spectral bands (NIR, SWIR1 and SWIR2). By that, we weight the aberration present in the respective spectrograph with the pupil intensity distribution. Table (1) shows the resulting errors of the ISRF. Particularly the ISRF shape is strongly affected, resulting in a variation of up to $0.67 \%$. Regarding the overall budget of $1.5 \%$, we conclude, that the fibre FF effects are a large contributor to the overall ISRF uncertainty budget and have to be mitigated.

Table 1: ISRF shape and centroid errors for non-uniform spectrograph pupil intensity distribution. The centroid shift is given in units of the spectral resolution.

\begin{tabular}{cccc} 
ISRF property & NIR $[780 \mathrm{~nm}]$ & SWIR1 $[1600 \mathrm{~nm}]$ & SWIR2 $[2000 \mathrm{~nm}]$ \\
\hline Shape error & $0.67 \%$ & $0.38 \%$ & $0.39 \%$ \\
Centroid shift (in $\Delta \lambda)$ & $0.24 \mathrm{pm}$ & $0.73 \mathrm{pm}$ & $0.93 \mathrm{pm}$ \\
\hline
\end{tabular}

In order to recover the FF uniformity, we investigated a technique to induce appropriate mechanical stress on the fibre. By slightly bending the fibre at small radii, the mode to mode coupling inside the fibre is increased. Fig. (6) shows the recovery of the FF homogeneity for a high contrast non-uniform scene. Repeating the raytracing 
method to determine the remaining errors yields negligible contribution for ISRF stability budget due to the fibre FF effect.

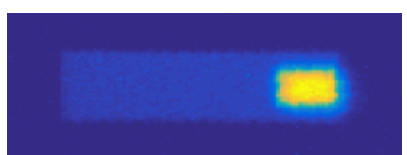

(a) Fibre input illumination

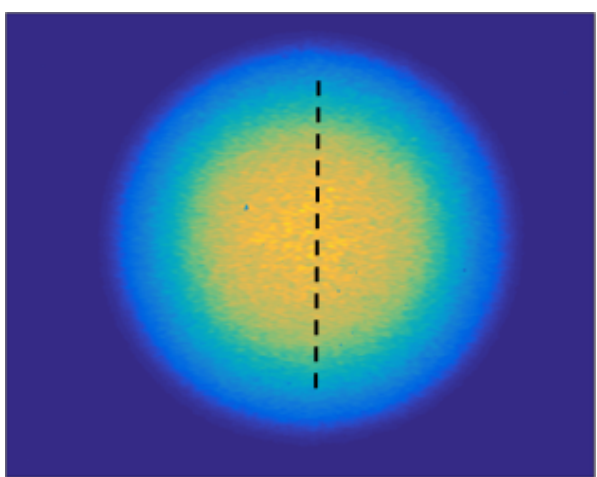

(b) Fibre FF in homogenized configuration

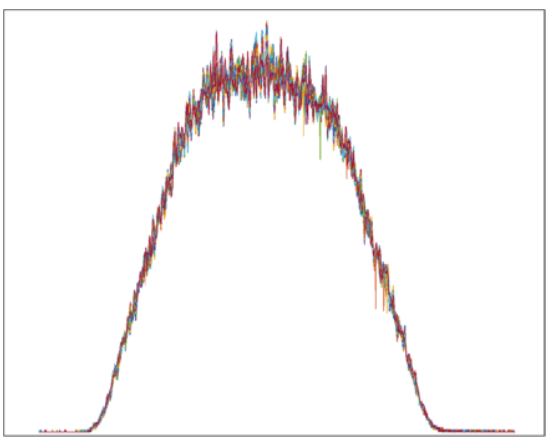

(c) Fibre FF cross section

Figure 6: By applying appropriate mechanical stress to the fibre, the homogeneity of the FF intensity distribution can be recovered.

We conclude, that using a sufficiently long fibre for a 2DSH is a necessary condition for efficiently homogenizing the fibre NF output. Further, it is essential to stimulate the mode-to-mode coupling inside the fibre to retrieve a homogeneous $\mathrm{FF}$ output. We investigated and tested a method to recover the FF homogeneity by applying appropriate stress on the fibre by means of small bending radii. For practical reasons, this is only possible for sufficiently long fibres. The concept for efficient NF and FF scrambling performance of a fibre-based 2DSH was filed in a provisional patent application by Airbus (EP 20 305786.4).

\section{FOCAL RATIO DEGRADATION (FRD)}

An important quantity of optical fibres it the so-called focal ratio degradation (FRD), which describes a degradation of the input $F \#$ into the fibre with respect to the output $F \#$. Depending on the sizing of the spectrograph optics, this will lead to a loss of signal and will therefore affect the system SNR. We tested the FRD losses for the $100 \mathrm{~cm}$ in a bended and straight configuration in the NIR and SWIR wavelength band. In the Airbus model of the CO2M A/B1 study, the telescope pupil $\left.\left(F_{t e l} \#=3.28\right)\right)$ is oversized with respect to the spectrograph pupil (see Fig. (7)). Therefore, we quantify the losses due to FRD in the following way: we sum up all of the output intensity after the fibre (red circle) and distribute it uniformly in the theoretical telescope pupil shape. Then we cut out the spectrograph pupil of our model (black rectangle) and take the ratio of the theoretical homogeneous pupil case and the real pupil intensity distribution after the fibre, which we define as the FRD losses. The results are summarized in Tab. (2). We observe a maximum transmission loss of $8.8 \%$ in the SWIR wavelength band. The applied mechanical stress in the bended fibre configuration has no significant impact on the FRD and therefore seems feasible in terms of radiometric losses. We want to emphasise, that these results are specifically linked to the assumed spectrograph pupil stop shape in our model.

Table 2: Radiometric losses due to FRD for a $100 \mathrm{~cm}$ long fibre in several mount and stress conditions.

\begin{tabular}{ccc} 
Fibre configuration & NIR $[780 \mathrm{~nm}]$ & SWIR $[1550 \mathrm{~nm}]$ \\
\hline Straight fibre & $6.0 \% \pm 1.0 \%$ & $8.8 \% \pm 0.19 \%$ \\
Bended fibre & $6.75 \% \pm 0.14 \%$ & $8.0 \% \pm 1.40 \%$ \\
\hline
\end{tabular}




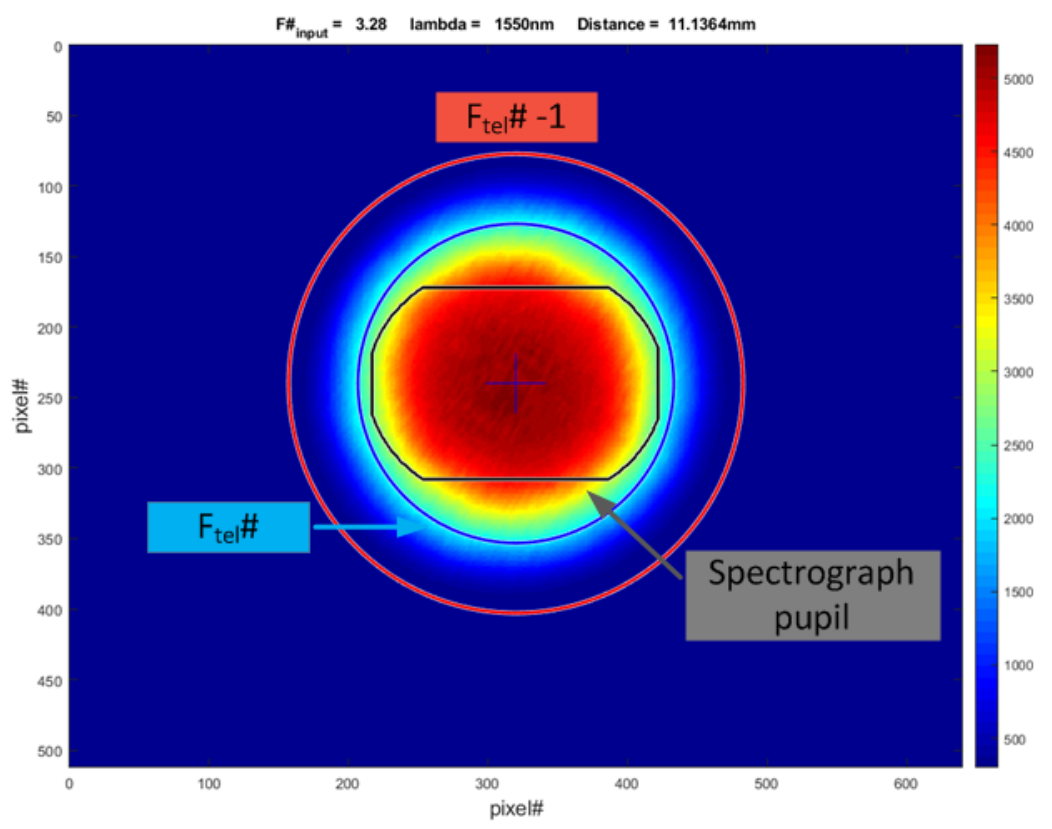

Figure 7: Typical fibre output in the FF for a homogeneous input. The black shape is the spectrograph pupil aperture baseline which is a rectangle with cut corners. The blue circle is the telescope pupil corresponding to an $\mathrm{F} \#$ of 3.28. The larger red circle $(\mathrm{F} \#=2.28)$ serves as a summation area in order to take possible FRD broadening into account.

\section{CONCLUSION}

Accurate atmospheric composition measurements like the CO2 Monitoring Mission require a stable ISRF, which needs to be insensitive to heterogeneous Earth scenes. The obtained experimental results in this paper yield the conclusion, that several evaluation parameters have to be taken into account for efficiently homogenizing the slit- and spectrograph illumination when using a 2DSH based on multimode fibres. The fibre length has to be sufficiently long to provide strong mode-to-mode coupling and thereby to homogenize the fibre NF output. Contrary to classical slits, a fibre-fed slit induces scene dependent spectrograph illuminations. Combined with the geometrical optical aberrations present in the spectrograph, this leads to another source of ISRF instability besides the slit illumination. Depending on the strength and type of aberrations, the error may become a high contributor to the global ISRF stability budget. We propose a solution to recover the FF uniformity by inducing appropriate mechanical stress by means of small bending radii applied to the fibre. Finally, we calculated the radiometric losses due to FRD which are dependent on the input $F \#$, stress induced on the fibre and wavelength. The results indicate that the spectral stability goals of the CO2M mission can be met under the proposed 2DSH solution.

\section{REFERENCES}

[1] Voors, R., Dobber, M., Dirksen, R., and Levelt, P., "Method of calibration to correct for cloud-induced wavelength shifts in the aura satellite's ozone monitoring instrument," Appl. Opt. 45, 3652-3658 (May 2006).

[2] Noël, S., Bramstedt, K., Bovensmann, H., Gerilowski, K., Burrows, J. P., Standfuss, C., Dufour, E., and Veihelmann, B., "Quantification and mitigation of the impact of scene inhomogeneity on sentinel-4 uvn uv-vis retrievals," Atmospheric Measurement Techniques 5(6), 1319-1331 (2012).

[3] Hu, H., Hasekamp, O., Butz, A., Galli, A., Landgraf, J., Aan de Brugh, J., Borsdorff, T., Scheepmaker, R., and Aben, I., "The operational methane retrieval algorithm for tropomi," Atmospheric Measurement Techniques 9(11), 5423-5440 (2016). 
[4] Landgraf, J., aan de Brugh, J., Scheepmaker, R., Borsdorff, T., Hu, H., Houweling, S., Butz, A., Aben, I., and Hasekamp, O., "Carbon monoxide total column retrievals from tropomi shortwave infrared measurements," Atmospheric Measurement Techniques 9(10), 4955-4975 (2016).

[5] Meister, C., Bauer, M., Keim, C., and Irizar, J., "Sentinel-5/uvns instrument: the principle ability of a slit homogenizer to reduce scene contrast for earth observation spectrometer," in [SPIE Proceedings Vol.10423, Sensors, Systems, and Next-Generation Satellites XXI; 104231E], (2017).

[6] Caron, J., Kruizinga, B., and Vink, R., "Slit homogenizers for Earth observation spectrometers: overview on performance, present and future designs," in [International Conference on Space Optics - ICSO 2018], Sodnik, Z., Karafolas, N., and Cugny, B., eds., 11180, 402 - 417, International Society for Optics and Photonics, SPIE (2019). 